\title{
Variational Solution for the Admittance of a Long Cylindrical Antenna
}

\author{
R. A. Hurd \\ Contribution from the Radio and Electrical Engineering Division, National Research Council, \\ Ottawa, Canada
}

(Received September 30, 1963)

\begin{abstract}
A variational expression for the admittance of a hollow cvlindrical antenna fed at the center has been obtained. Contrary to normal practice, the integral equation and the resulting variational expression have as the unknown function, the tangential electric field in the tubular region extending from the antenna ends to $\pm \infty$. A plausible trial field is chosen and the relevant integrals are evaluated asymptotically for long, thin antennas. Some numerical results are given which show reasonable agreement with the experimental values of Iizuka et al. [1963], and also with the theoretical results of Wu [1961].
\end{abstract}

\section{Introduction}

Recently there has been considerable interest shown in the admittance of long cylindrical antennas. Vainshtein [1959], by assuming that the current consisted of incoming and outgoing waves with amplitudes which were slowly varying functions of position, reduced the problem to the solution of Volterra integral equations which could be solved exactly. Another approach was used by Wu [1961] who approximated a long antenna by a semiinfinite one. The latter problem was then solved by Wiener-Hopf techniques. Extensive tables of numerical values were presented by $\mathrm{Wu}$. Quite a different method was used by Chen and Keller [1962], who assumed the current to be composed of a wave emanating from the feedpoint plus waves reflected from the ends. The current reflection coefficient at the end of a tube was known from previous work. With a knowledge of this quantity the authors were able to obtain the admittance without formally solving an integral equation. Essentially the same method was used earlier by Hallén [195், 1962]. Studies on the infinite antenna were carried out by Hallén [1948], Papas [1948], Duncan [1962], and Kunz [1963]. Measurements on long antennas have been made by Tizuka et al. [1963], whose results agree quite well with the theoretical values of $\mathrm{Wu}$ [1961]. Variational nethods were applied to the antenna problem by Storer [1950] and by Tai [1950], who obtained variational expressions for the impedance in terms of the unknown current distribution on the antenna. Such a formulation is expected to be most accurate for short antennas. To obtain an integral equation, and from it a variational expression whose approximate solution is most accurate for long antennas, one should use the tangential electric field in the "aperture" as the unknown function. ${ }^{1}$ Here the aperture is understood to be the tubular extension reaching to $\pm \infty$ from each end of the antenna. To the writer's knowledge an integral equation containing the electric field as unknown has never been used, and this approach forms the substance of this paper.

\section{Integral Equation and Variational Expression}

The antenna is assumed to be a thin-walled, perfectly conducting tube occupying the region $\rho=a,-h \leq z \leq h$, of the $(\rho, \phi, z)$ cylindrical coordinate system. At $z=0$ the antenna is driven by a voltage $V$ applied across an infinitesimal circumferential gap. The only electric field component in the gap is given by

$$
E_{z}=-V \delta(z) e^{i \omega t}
$$

The space surrounding the antenna is divided into two regions, denoted " 1 " for $\rho<a$ and " 2 " for $\rho>a$. Field components in these regions will henceforth be distinguished by appropriate superscripts.

It is assumed that all field components are derivable from the z-components of electric hertz vectors. In either region, after dropping the time-dependence factor,

$$
\Pi_{z}^{(i)}=\int_{-\infty}^{\infty} A_{i}(\alpha) Z_{i}(\beta \rho) e^{i \alpha z} d \alpha, \quad i=1,2 ;
$$

1 This seems a reasonable extension of a statement of Levine [1955] p. 38, who was concerned with diffraction by a circular aperture. I am indebted to R. F. Millar for supplying this reference, and for a helpful discussion of this point. 
where $\beta^{2}=k^{2}-\alpha^{2}$, the $A_{i}(\alpha)$ are amplitude functions, and the $Z_{i}$ are Bessel functions appropriate to the region. The contour of integration is assumed to be indented downward around the branch point $\alpha=-k$, and upward at $\alpha=k$. From (2), the tangential electric and magnetic field components are easily obtained:

$$
\begin{gathered}
E_{z}^{(i)}=\int_{-\infty}^{\infty} \beta^{2} A_{i}(\alpha) Z_{i}(\beta \rho) e^{i \alpha z} d \alpha, \\
H_{\varphi}^{(i)}=-i \omega \epsilon_{0} \int_{-\infty}^{\infty} \beta A_{i}(\alpha) Z_{i}^{\prime}(\beta \rho) e^{i \alpha z} d \alpha,
\end{gathered}
$$

the dash on the Bessel function indicating derivative with respect to the argument. At $\rho=a, E_{z}$ must be continuous for all $z$, thus

$$
A_{1}(\alpha) Z_{1}(\beta a)=A_{2}(\alpha) Z_{2}(\beta a) .
$$

Also $E_{z}=0$ for $\rho=a,-h \leq z \leq h$, with the exception of $z=0$; so that, upon taking the inverse Fourier transform of (3) and using (1),

$$
2 \pi \beta^{2} A_{i}(\alpha) Z_{i}(\beta a)=\int_{|z|>h} e^{-i \alpha z} E_{z}(z) d z-V .
$$

In the region $z>h, \rho=a, H_{\phi}$ must be continuous, hence from (4)

$\int_{-\infty}^{\infty} \beta e^{i \alpha z}\left[A_{1} Z_{1}^{\prime}(\beta a)-A_{2} Z_{2}^{\prime}(\beta a)\right] d \alpha=0, \quad|z|>h$.

For $|z|<h, \rho=a$ the total current $I_{t}$ on the antenna is given by $I_{t}=-2 \pi a\left[H_{\phi}^{(1)}-H_{\phi}^{(2)}\right]$, which from (4) is $I_{t}(z)=2 \pi i \omega \epsilon_{0} a \int_{-\infty}^{\infty} \beta e^{i \alpha z}\left[A_{1}(\alpha) Z_{1}^{\prime}(\beta a)-A_{2}(\alpha) Z_{2}^{\prime}(\beta a)\right] d \alpha$, $|z|<h$.

Use is made now of (5) and (6) to remove $A_{1}(\alpha)$ and $A_{2}(\alpha)$ from (7) and (8) with the result

$$
\begin{aligned}
\int_{-\infty}^{\infty} \frac{e^{i \alpha z} d \alpha}{\beta^{2} J_{0}(\beta a) H_{0}^{(2)}(\beta a)} & \\
\left\{\int_{\left|z^{\prime}\right|>h} e^{-i \alpha z^{\prime}} E_{z}\left(z^{\prime}\right) d z^{\prime}-V\right\} & =0, \quad|z|>h \\
& =\frac{-\pi I_{t}(z)}{2 \omega \epsilon_{0}} \\
& |z|<h ;
\end{aligned}
$$

where $Z_{1}$ has been replaced by $J_{0}$ and $Z_{2}$ by $H_{0}^{(2)}$, and the Wronskian relation $J_{0} H_{0}^{(2) \prime}-H_{0}^{(2)} J_{0}^{\prime}=$ $-2 i /(\pi \beta a)$ has been used. Interchanging the order of integration and writing $E(z)$ for $E_{z}(z)$ yields the integral equation for $E(z)$

$$
\int_{\left|z^{\prime}\right|>h} E\left(z^{\prime}\right) K\left(z-z^{\prime}\right) d z^{\prime}=V K(z), \quad|z|>h,
$$

and the relation

$\int_{\left|z^{\prime}\right|>h} E\left(z^{\prime}\right) K\left(z-z^{\prime}\right) d z^{\prime}=V K(z)-\frac{\pi I_{t}(z)}{2 \omega \epsilon_{0}}, \quad|z|<h ;$

where

$$
K(z)=\int_{-\infty}^{\infty} \frac{e^{i \alpha z} d \alpha}{\beta^{2} J_{0}(\beta a) H_{0}^{(2)}(\beta a)}
$$

To obtain a variational expression, (11) is first multiplied by $E(z)$ and integrated with respect to $z$ over $|z|>h$, to give

$$
\begin{aligned}
\iint_{|z|,\left|z^{\prime}\right|>h} E(z) E\left(z^{\prime}\right) K\left(z-z^{\prime}\right) d z d z^{\prime} & \\
& =V \int_{|z|>h} E(z) K(z) d z .
\end{aligned}
$$

If $z$ is allowed to approach zero in (12) one obtains, since $K(-z)=K(z)$,

$$
\int_{|z|>h} E(z) K(z) d z=V K(0)-\frac{\pi I_{t}(0)}{2 \omega \epsilon_{0}} .
$$

Equation (14) now is divided by $\left[\int E(z) K(z) d z\right]^{2}$ and (15) used to introduce $I_{t}(0)$, giving

$\frac{\pi I_{t}(0)}{2 \omega \epsilon_{0} V}-K(0)=-\frac{\left[\int_{|z|>h} E(z) K(z) d z\right]^{2}}{\iint_{|z|,\left|z^{\prime}\right|>h} E(z) E\left(z^{\prime}\right) K\left(z-z^{\prime}\right) d z d z^{\prime}}$.

This is a variational expression for the quantity on the left. The proof of this is omitted, but follows the standard procedure. An interpretation of the quantity $K(0)$ is now required. This is readily obtained from (12). If $h \rightarrow \infty$ the integral on the left tends to zero, so that

$$
K(z)=\pi I_{t}^{\infty}(z) / 2 \omega \epsilon_{0} V
$$

where $I_{t}^{\infty}$ is the total current on an infinite antenna. If $z$ approaches zero, then

$$
K(0)=\pi Y_{t}^{\infty} / 2 \omega \epsilon_{0},
$$

with $Y_{t}^{\infty}$ the total admittance at the feed point of an infinite antenna. Then (16) becomes

$$
Y_{t}-Y_{t}^{\infty}=-\frac{2 \omega \epsilon_{0}}{\pi} \frac{\left[\int \mathcal{E}(z) K(z) d z\right]^{2}}{\int \mathcal{S} E(z) E\left(z^{\prime}\right) K\left(z-z^{\prime}\right) d z d z^{\prime}} .
$$

This is the variational expression in its final form. It relates the admittance of a finite but long antenna to that of the infinite one. The latter has been the subject of considerable research, to which Papas [1948], Hallén [1948], Duncan [1962], and Kunz [1963] have all contributed. 
A remark about (19) is in order. Although the admittances given are total ones; that is, they include the effects of currents inside the tube, the expression (19) is approximately true also for the external admittances (called $\bar{Y}$ and $Y^{\infty}$ ), when the tube diameter is reasonably small. This follows because the interior currents are excited by evanescent waveguide modes which are rapidly attenuated by distance, and which consequently are hardly affected by the antenna ends. Thus the interior currents are almost equal for infinite and long antennas and hence cancel in (19).

\section{Evaluation of the Variational Expression}

The problem now arises of choosing a suitable field distribution $E(z)$ to use in (19). There are two obvious requirements which this function should satisfy: it must behave like $e^{-i k|z|} /|z|^{2}$ for large $|z|$, and it should vary as $[|z|-h]^{-1 / 2}$ near $|z|=h$. Both can be achieved by taking

$$
E(z)=|z|^{-1}\left(z^{2}-h^{2}\right)^{-1 / 2} \exp (-i k|z|) .
$$

A constant multiplier might have been included in (20), but was omitted because the variational expression is independent of this term.

When (20) is inserted in (19), integrals of the following kinds arise:

$$
\begin{gathered}
I_{1}=\iint_{|z|,\left|z^{\prime}\right|>h} \frac{\exp \left[-i k\left(|z|+\left|z^{\prime}\right|\right)\right] K\left(z-z^{\prime}\right) d z d z^{\prime}}{\left(z^{2}-h^{2}\right)^{1 / 2}\left(z^{\prime 2}-h^{2}\right)^{1 / 2}\left|z z^{\prime}\right|}, \\
I_{2}=\int_{|z|>h} \frac{\exp (-i k|z|) K(z) d z}{\left(z^{2}-h^{2}\right)^{1 / 2}|z|} .
\end{gathered}
$$

The approximate evaluation of these integrals is carried out in the appendix, under the assumptions that the radius is small in terms of wavelength and that the length of the antenna is great. The results are:

$$
\begin{gathered}
I_{1} \simeq-\frac{i \pi^{3}}{2 k^{2} h^{3} L_{2}} \exp (-4 i k h)+\frac{i \pi^{2}}{k^{2} h^{3} L_{0}} \exp (-2 i k h), \\
I_{2} \simeq\left(\pi^{5} / k^{3} h^{3}\right)^{1 / 2} L_{1}^{-1} \exp (-2 i k h-i \pi / 4),
\end{gathered}
$$

where

$$
\begin{aligned}
& L_{0}=\ln \left(\frac{1}{2} i \Gamma k a\right) \\
& L_{1}=\ln \left(-2 i h / \Gamma k a^{2}\right) \\
& L_{2}=\ln \left(-4 i h / \Gamma k a^{2}\right),
\end{aligned}
$$

and $\Gamma=1.781 \ldots$. If the results (23) and (24) are used in (19), the following expression for the admittance $Y-Y^{\infty}$ is obtained

$$
Y-Y^{\infty}=\frac{-4 \pi Y_{0} L_{2}}{L_{1}^{2}\left[1-\left(2 L_{2} / \pi L_{\jmath}\right) \exp (2 i k h)\right]}
$$

where $Y_{0}=(120 \pi)^{-1}$ mhos is the admittance of free space.
Expression (25) closely resembles, in form, Halléa' result [1962, p. 485]. Apart from a difference in the argument in one of the logarithms, which is of the same magnitude as higher order terms omitted in the expansion of $Y-Y^{\infty}$, the only difference is the replacement of a factor $2 / \pi$ in the denominator of (25) by a factor $\frac{1}{2}$ in Hallén's work. This difference has quite a large effect near resonance.

\section{Discussion of Results}

To compare (25) with the results of other workers, an expression for $Y^{\infty}$ is needed. $\mathbf{A}$ difficulty is that $Y^{\infty}$ turns out to be infinite, as noted by Infeld [1947] and others. This point has been discussed by Duncan [1962], who proposes a "smoothed" form of the admittance for an infinite antenna. In the present paper values interpolated from Duncan's table 3 are used. Two different radii are employed: $a / \lambda=0.0085$ and $a / \lambda=0.001191$. The values of $Y^{\infty}$ from Duncan's paper corresponding to these were found to be $0.00300+0.00187 i$ and $0.00200+0.00084 i$ mhos, respectively.

The results of the calculation based on (25) and carried out for three different ranges of antenna lengths are shown in figures 1, 2, and 3. Some experimental data for long antennas have been given by Tizuka et al. [1963], but owing to the smallness of their graphs, it is not easy to compare their results directly with calculations based on (25). However, their curves follow closely Wu's [1961] theoretical values, which are tabulated, and which have been included in figures 1,2 , and 3 . It will be observed that the present results for the range $51.0 \leq k h \leq 52.6$ (fig. 3) are displaced to the right of Wu's, which in turn are on the right of the experimental curves of

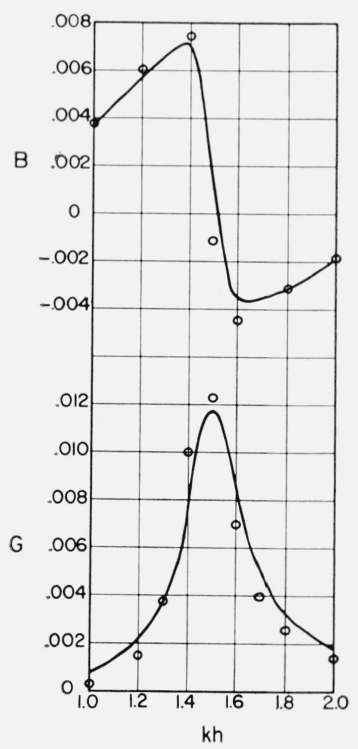

Figure 1. Admittance of a cylindrical antenna, $\mathrm{a} / \lambda=0.001191$. $1.0 \leq \mathrm{kh} \leq 2.0$.

Dots indicate Wu's theoretical values. 


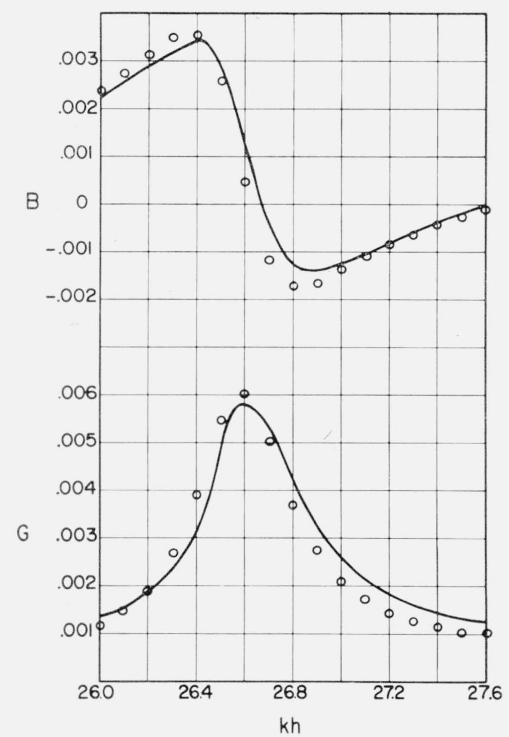

Figure 2. Admittance of a cylindrical antenna, $\mathrm{a} / \mathrm{\lambda}=0.001191$, $26.0 \leq \mathrm{kh} \leq 27.6$.

Wots indicate Wu's theoretical values.

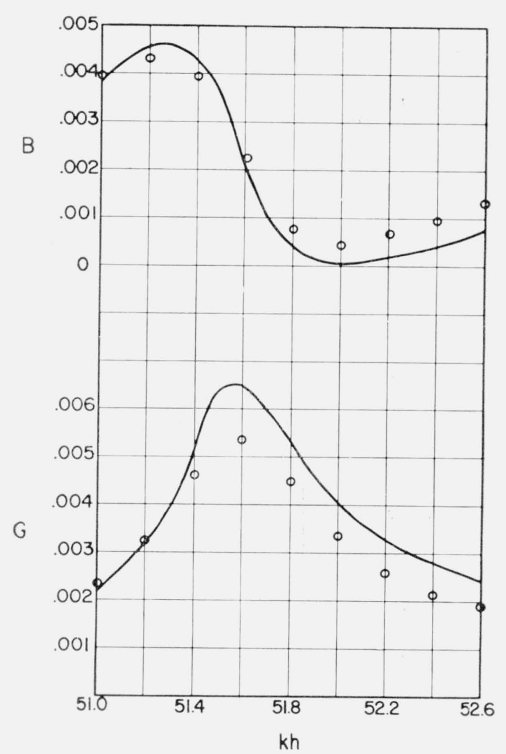

Figure 3. Admittance of a cylindrical antenna, $\mathrm{a} / \lambda=0.0085$, $51.0 \leq \mathrm{kh} \leq 52.6$.

Dots indicate Wu's theoretical values.

Iizuka et al. [1963]. In addition, the present $\mathrm{G}_{\max }$ exceeds Tizuka's by about 20 percent. Figure 2, for $26.0 \leq k h \leq 27.6$, reveals closer agreement between Wu's and the present results. Somewhat surprisingly the agreement over the range $1.0 \leq k h \leq 2.0$ (fig. 1) is not materially worse than for the longer range. It is not possible to compare the last two curves with experiment at the present time.

\section{Conclusion}

In conclusion, a simple variational solution for the admittance of a cylindrical antenna has been obtained, involving the longitudinal electric field in the extension of the antenna as unknown. This formulation is most accurate for long antennas, in contrast with the variational method in terms of current, which is more suitable for short antennas. For long antennas it is easier to choose a plausible electric field than a current, since the latter involves waves in two directions with an unknown reflection coefficient. Using a reasonable trial field, the first term of an asymptotic series for the admittance is obtained. This gives fairly good agreement with experiment and with Wu's theory [1961]. More accuracy will be obtained if extra terms in the asymptotic development are included, provided the antenna is sufficiently long.

The author thanks Mrs. M. Steen for carefully doing the calculations, and E. V. Jull for reading and criticising the manuscript.

\section{Appendix. Evaluation of the Integrals}

The integral $I_{1}$ of (21) is first considered. By halving the ranges of integration and taking account of the evenness of $K(z), I_{1}$ can be written in the form

$$
\begin{aligned}
I_{1}=2 \int_{0}^{\infty} \frac{d \alpha}{\beta^{2} J_{0}(\beta a) H_{0}^{(2)}(\beta a)} \\
\left\{\int_{h}^{\infty} \frac{\left(e^{i \alpha z}+e^{-i \alpha z}\right) e^{-i k z} d z}{z\left(z^{2}-h^{2}\right)^{1 / 2}}\right\} .^{2}
\end{aligned}
$$

The term in braces can be expressed in terms of Hankel functions through the relation

$$
H_{0}^{(2)}(h x)=\frac{2 i}{\pi} \int_{h}^{\infty} \frac{e^{-i x z} d z}{\left(z^{2}-h^{2}\right)^{1 / 2}}
$$

giving approximately, for large $h$

$$
\begin{aligned}
I_{1}=-\frac{\pi^{2}}{2 h^{2}} \int_{0}^{\infty} \frac{d \alpha}{\beta^{2} J_{0}(\beta a) H_{0}^{(2)}(\beta a)} & \left\{H_{0}^{(2)}[(\alpha+k) h]\right. \\
& \left.+H_{0}^{(2)}[(k-\alpha) h]\right\}^{2} .
\end{aligned}
$$

For reasonably large $h, H_{0}[(\alpha+k) h]$ can be replaced by its asymptotic value. It is desirable to approximate the other Hankel function the same way, but it is not immediately clear that this can be done, since its argument is small near $\alpha=k$. However, the contour can be deformed into the first quadrant away from $\alpha=k$ without crossing any singularities, ${ }^{2}$ hence on the new contour the approximation is valid if $h$ is sufficiently large. The final step consists of deforming back to the original contour with

2 The poles of the integrand are the zeros of $J_{0}(\beta a)$ which all lie on the contour $\operatorname{Im}(\boldsymbol{\beta})=0$. By virtue of the time dependence chosen these lie in the $2 \mathrm{~d}$ and $4 \mathrm{th}$ quadrants. 
the result

$$
\begin{aligned}
I_{1} & =\frac{-\pi i}{h^{3}} e^{-2 i k h} \int_{0}^{\infty} \frac{d \alpha}{\beta^{2} J_{0}(\beta a) H_{0}^{(2)}(\beta a)}\left\{\frac{e^{-2 i \alpha h}}{k+\alpha}+\frac{e^{2 i \alpha h}}{k-\alpha}+\frac{2}{\beta}\right\} \\
& \equiv\left(-\pi i / h^{3}\right) e^{-2 i k h}\left[I_{11}+I_{12}+I_{13}\right] . \quad(28)
\end{aligned}
$$

Integrals of the type $I_{11}$ take their values mainly from the neighbourhood of $\alpha=k$; so that

$$
I_{11} \simeq \frac{\pi i}{4 k} \int_{0}^{\infty} \frac{e^{-2 i \alpha h} d \alpha}{\beta^{2} \ln \left(\frac{1}{2} \Gamma \beta a\right)},
$$

where the Bessel functions have been replaced by their small argument approximations. The integral in (29) has for its leading term

$$
I_{11} \simeq \frac{\pi^{2}}{2 k^{2}} \frac{e^{-2 i k h}}{L_{2}}
$$

[Hallén 1948].

The integral $I_{12}$, though of similar appearance, takes quite a different form. Since $e^{2 i \alpha h} \rightarrow 0$ for $\operatorname{Im}(\alpha) \rightarrow \infty$, the contour can be moved one along the positive imagmary axis:

$I_{12}=i \int_{0}^{\infty} \frac{e^{-2 \zeta h} d \zeta}{(k-i \zeta)\left(k^{2}+\zeta^{2}\right) J_{0}\left[a\left(k^{2}+\zeta^{2}\right)^{1 / 2}\right] H_{0}^{(2)}\left[a\left(k^{2}+\zeta^{2}\right)^{1 / 2}\right]}$.

Watson's lemma (see Jeffreys and Jeffreys [1962], p. 501) can now be applied, with the result $I_{12}=$ $0(1 / h)$, so that $I_{12}$, to the first approximation, is negligible compared with $I_{11}$. Strictly speaking, the poles which lie near, and in the absence of loss on, the path of integration should be accounted for, but if $a$ is small, they occur for large values of $\zeta$ and thus contribute very little to $I_{12}$.

There remains $I_{13}$. The contour of integration is first changed to the positıve imaginary axis, with the result

$$
I_{13}=2 i \int_{0}^{\infty} \frac{d \zeta}{\left(k^{2}+\zeta^{2}\right)^{3 / 2} J_{0}\left[a\left(k^{2}+\zeta^{2}\right)^{1 / 2}\right] H_{0}^{(2)}\left[a\left(h^{2}+\zeta^{2}\right)^{1 / 2}\right]} .
$$

The range of integration is now split at $\zeta=b$, so that in $(0, b)$ the small argument approximation to the Bessel function applies. The remaining integral on $(b, \infty)$, being of order $1 / b^{2}$ and hence $0\left(a^{2}\right)$, is neglected. Then

$$
I_{13} \simeq-\pi \int_{0}^{b} \frac{d \zeta}{\left(h^{2}+\zeta^{2}\right)^{3 / 2} \ln \left[\frac{1}{2} i \Gamma a\left(k^{2}+\zeta^{2}\right)^{1 / 2}\right]} \cdot
$$

In (33) the logarithmic term is a slowly varying function, and without large error $\zeta$ in it can be replaced by a constant lying in the range $(0, b)$. The value chosen is not very important since any error is of the order of the terms omitted in the expansion.
It is reasonable to take $\zeta=0$, however, since the term $\left(k^{2}+\zeta^{2}\right)$ is smallest there. The integration remaining is elementary, giving

$$
I_{13} \simeq-\pi b / k^{2} L_{0}\left(k^{2}+b^{2}\right)^{1 / 2} .
$$

Recalling that $b$ is large, approximately

$$
I_{13}=-\pi / k^{2} L_{0} \text {. }
$$

When the terms are gathered together, the final result is

$$
I_{1} \simeq \frac{-\pi^{3} i e^{-4 i k h}}{2 k^{2} h^{3} L_{2}}+\frac{\pi^{2} i e^{-2 i k h}}{k^{2} h^{3} L_{0}}
$$

The integral $I_{2}$ given in (22) is now evaluated. Following the course outlined for $I_{1}$, the integral $I_{2}$ becomes

$$
\begin{aligned}
I_{2}=-\frac{i \pi}{h} \int_{0}^{\infty} \frac{d \alpha}{\beta^{2} J_{0}(\beta a) H_{0}^{(2)}(\beta a)}\{ & H_{0}^{(2)}[(k+\alpha) h] \\
& \left.+H_{0}^{(2)}[(k-\alpha) h]\right\}
\end{aligned}
$$

which, as before, can be further simplified to

$$
\begin{aligned}
I_{2}=-i\left(\frac{2 \pi}{h^{3}}\right)^{1 / 2} e^{i\left(\frac{\pi}{4}-k h\right)} \int_{0}^{\infty} \frac{d \alpha}{\beta^{2} J_{0}(\beta a) H_{0}^{(2)}(\beta a)} \\
\\
\left\{\frac{e^{i \alpha h}}{(k-\alpha)^{1 / 2}}+\frac{e^{-i \alpha h}}{(k+\alpha)^{1 / 2}}\right\} .
\end{aligned}
$$

The terms in (37) are similar to $I_{11}$ and $I_{12}$ in (28), and may be handled in like manner, with the result

$$
I_{2} \simeq\left(\pi^{5} / k^{3} h^{3}\right)^{1 / 2} L_{1}^{-1} \exp (-2 i k h-i \pi / 4) .
$$

\section{References}

Chen, Y. M., and J. B. Keller [1962], Current on and input impedance of a cylindrical antenna, J. Res. NBS 66D (Radio Prop.) No. 1, 15-21.

Duncan, R. H. [1962], Theory of the infinite cylindrical antenna including the feedpoint singularity in antenna current, J. Res. NBS 66D (Radio Prop.) No. 2, 181-188.

Hallén, E. [1948], Properties of a long antenna, J. Appl. Phys. 19, 1140-1147.

Hallén, E. [1961], Exact treatment of current wave reflection at the end of a tube-shaped cylindrical antenna, IRE Trans. Antennas Propagation, AP-4, 479-491.

Hallén, E. [1962], Electromagnetic Theory (Chapman and Hall, London).

Iizuka, K., R. W. P. King, and S. Prasad [1963], The admittance of very long cylindrical antenna, Proc. IEE 110, No. $2,303-309$.

Infeld, L. [1947], The influence of the gap in the theory of antennas, Quart. Appl. Math. 5, 113.

Jeffreys, H., and B. Jeffreys [1956], Methods of Mathematical Physics, p. 501 (Cambridge University Press).

Kunz, K. S. [1963], Asymptotic behavior of the current on an infinite cylindrical antenna, J. Res. NBS 6zD (Rad Prop.) No. 4, 417-431. 
Levine, H. [1955], Diffraction by a circular aperture at high frequencies, New York University, Division of Electromagnetic Research, Research Rpt. No. EM-84.

Papas, C. H. [1948], On the infinitely long cylindrical antenna, Cruft Lab. Tech. Rpt. No. 58, Sept. 10.

Storer, J. E. [1950], A variational solution to the problem of the symmetrical cylindrical antenna, Cruft Lab. Tech. Rpt. No. 101, Feb. 10.

Tai, C. T. [1950], A variational solution to the problem of cylindrical antennas, Stanford Research Institute Tech. Rpt. No. 12, SRI Project No. 188.
Vainshtein, L. A. [1959], Waves of current in a thin cylindrica ] conductor: I. Currents and impedance of a transmitting antenna, J. Tech. Phys. USSR 29, No. 6, English Translation, Soviet Phys. Tech. Phys. 4, 601-615, 1959.

Wu, T. T. [1961], Theory of the dipole antenna and the twowire transmission line, J. Math. Phys. 2, No. 4, 55-574.

(Paper 68D3-347) 